\title{
Argon laser photocoagulation in the treatment of central serous retinopathy
}

\author{
PETER LEAVER AND CHRISTINE WILLIAMS \\ From the Retinal Diagnostic Department, Moorfields Eye Hospital, City Road, London EC1V 2PD
}

SUMMARY In a prospective randomised trial of argon laser photocoagulation in the management of central serous retinopathy it was confirmed that this treatment hastens resolution of the serous detachment. No evidence was found to suggest that treatment influences the final visual outcome in eyes with initial visual acuity of $6 / 12$ or better.

Although the pathogenesis, clinical features, and natural history of central serous retinopathy (CSR) have been more clearly defined in recent years, the indications for treatment are not precisely established. While many forms of therapy have been advocated, the benign nature of the condition and its tendency to recover spontaneously make their critical evaluation difficult. The predilection of CSR for young males, its tendency to persist for long periods, and the high recurrence rate make effective treatment desirable.

The ability to demonstrate the source of subretinal fluid in CSR by fluorescein fundus angiography (Maumenee, 1965; Gass, 1967), together with development of more sophisticated photocoagulation techniques (Wessing, 1971), suggested that an effective method of treatment might be available. Recent studies have shown that photocoagulation causes rapid resolution of the serous retinal detachment with symptomatic recovery (Spalter, 1968; Peabody et al., 1968; Watzke et al., 1974; Nanjiani, 1977).

On the basis of clinical impression it is acknowledged that photocoagulation can be justified if corrected visual acuity is worse than 6/12 (Hogan, 1974) or 0.8 (Wessing, 1974), if it is a second attack in the eye in question, and if there has been a permanent loss of acuity in the fellow eye from central serous retinopathy (Gass, 1974a). If the corrected visual acuity is good, some clinicians do not treat the condition (Hogan, 1974; Schoch, 1974; Klein et al., 1974), while others recommend therapy if there are morphological changes in the detached retina, or if the symptoms interfere significantly with life (Wessing, 1974), though symptoms may persist after retinal reattachment and may never fully resolve (Gass, 1974b; Zweng, 1974).

Correspondence to P. K. Leaver, FRCS.
In the common situation when these indications are absent it has been suggested that photocoagulation can be justified 2 or 3 months (Amalric, 1974; Gass, 1974a; Nanjiani, 1977) after the onset of symptoms. Such a recommendation can be sustained only if it is shown that the length of detachment determines the final visual outcome. To test this we undertook a prospective randomised trial of treatment with argon laser photocoagulation to discover how the natural history of CSR is modified by treatment and the effect of this on the visual outcome.

Definition. Central serous retinopathy was defined as a localised serous detachment of the retina occurring at the posterior pole in young people, for which no predisposing cause could be found. Serous retinal detachments associated with other identifiable lesions, such as tumours, inflammation, or congenital pits of the optic disc and generalised disease of the retinal pigment epithelium (RPE), were excluded, although multiple RPE defects were frequently present at the posterior pole in both eyes.

\section{Patients and methods}

Sixty-seven consecutive patients referred to the Retinal Diagnostic Department at Moorfields Hospital, City Road, London, with CSR were entered in the study having satisfied the following criteria: (1) corrected visual acuity $6 / 12$ or better; (2) retina detached at macula; (3) RPE defects smaller than 1 disc diameter; (4) no symptomatic improvement since onset; (5) no subretinal exudates present; (6) no cystic retinal oedema present; (7) no associated ocular disease (e.g., drusen, congenital pit of the disc, generalised RPE dystrophy, etc.); (8) consent to participate in the study after explanation of aims and methods.

Patients were allocated to 4 groups, by drawing 
randomised cards from sealed envelopes, according to age (39 years or less; 40 years or more) and time of presentation (shorter or longer than 1 month after onset of symptoms). Half the eyes in each group were left untreated, and in the remainder treatment was carried out as soon as fluorescein angiograms were available. It was ruled that eyes in the untreated group in which corrected visual acuity fell to less than $6 / 12$ during the study or which developed cystic retinal oedema or subretinal exudates should be treated and classified as failed conservative management.

The Coherent Radiation 800 argon laser was used for treatment after fluorescein angiographic labelling of the leaking site. Direct treatment to the leaking spot was carried out with burns of 50 to $200 \mu \mathrm{m}$ in diameter, unless the leak was immediately subfoveal, when some 5-7 burns were applied within the lower margin of the serous retinal detachment ( 2 cases). Intensity and duration of the laser burns varied between 150 and 400 milliwatts and 20 and 200 milliseconds.

Patients were reviewed at approximately weekly intervals for 4 weeks and then at monthly intervals until symptoms improved, the retina flattened, and there was no identifiable leakage on fluorescein fundus angiography (FFA). Thereafter examinations were carried out at 3- and 6-monthly intervals.

Symptoms of central visual disturbance, in particular micropsia and metamorphopsia, were recorded at each visit, and the fundus was examined with the binocular indirect ophthalmoscope, slit lamp, and Hruby lens. Visual function was assessed by the best corrected Snellen visual acuity at each visit, while colour and fluorescein photography were undertaken in all cases at presentation and resolution and at other times when it was considered helpful. In 51 eyes the Farnsworth Munsell 100-hue test was carried out at each visit. Further investigations with the Amsler Chart, Nagel Anomaloscope, and electrodiagnostic studies were undertaken in some cases.

Of the 67 patients entered 4 had to be withdrawn, 3 because of failure to attend for follow-up examination and 1 who developed a disciform lesion. There were 53 males and 10 females, their ages ranging from 25 to 53 years with a mean of $40 \cdot 1$. The period of time between onset and presentation varied from 2 days to 35 weeks, with a mean of 5.6 weeks. Fifty-six of the patients were Caucasians, 9 were Asians and 2 Negroes.

\section{Results}

In all 67 eyes serous retinal detachment was associated with a demonstrable leak of fluorescein dye through the retinal pigment epithelium. In 35 patients $(49 \%)$ transmission defects in the RPE were also found or there was a clear history of similar attacks previously in the same eye.

\section{RECOVERY TIME}

Complete recovery as shown by resolution of symptoms, reattachment of the retina, and absence of dye leakage on FFA, occurred at a mean time of 6.2 weeks from entry into the treated group and 16.0 weeks into the control group $(P<0.001)$.

\section{VISUAL ACUITIES}

Snellen acuities were converted to a simple numerical scale from $1(6 / 4)$ to $5(6 / 12)$. The mean acuity on presentation was $4 \cdot 1$ for the control group and 3.9 for the treated group of patients and improved to 3.1 and 2.9 respectively at 6 months (Table 1 ). A significant difference between the visual acuities of the treated and untreated eyes occurred at 2 months after entry but at no other time. The mean visual acuity of the fellow eyes was $2 \cdot 8$ (Table 1 , Fig. 1). Nine patients failed to achieve a final acuity of $6 / 6$ or better. Six of these were in the control group and 3 were in the treated group; in all these cases the final acuity was 6/9. In no instance was it necessary to withdraw a patient from the trial because of a fall in visual acuity to less than $6 / 12$.

\section{HUE DISCRIMINATION}

At entry the mean of the 100-hue scores in the control group was 212 and in the treated group 282, improving to 137 and 156 respectively at 6 months. No significant difference between the mean scores of the 2 groups was found at any stage (Table 1). The mean 100-hue score of the fellow eyes was 90 .

Table 1 Change in the mean visual acuities and Farnsworth Munsell 100-hue scores in the treated and control groups from the time of entry into the trial. Visual acuity; $1-6 / 4,2-6 / 5,3-6 / 6,4-6 / 9,5-6 / 12$

\begin{tabular}{lllllll}
\hline $\begin{array}{l}\text { Interval } \\
\text { after entry } \\
\text { to trial }\end{array}$ & \multicolumn{2}{l}{ Snellen acuity } & \multicolumn{5}{c}{$F-M$ 100-hue } \\
\cline { 2 - 7 } & Controls & Treated & $P$ & Controls & Treated & $P$ \\
\hline On entry & 4.1 & 3.9 & 0.245 & 212 & 282 & 0.117 \\
2 weeks & 4.1 & 3.9 & 0.206 & 189 & 243 & 0.200 \\
4 weeks & 4.0 & 3.6 & 0.106 & 159 & 149 & 0.819 \\
2 months & 3.7 & 3.1 & 0.003 & 178 & 149 & 0.533 \\
3 months & 3.5 & 3.1 & 0.089 & 160 & 101 & 0.145 \\
6 months & 3.1 & 2.9 & 0.211 & 137 & 156 & 0.718 \\
$>6$ months & 3.2 & 2.9 & 0.167 & 154 & 132 & 0.621 \\
Fellow eyes & & 2.8 & & & 90 & \\
\hline
\end{tabular}




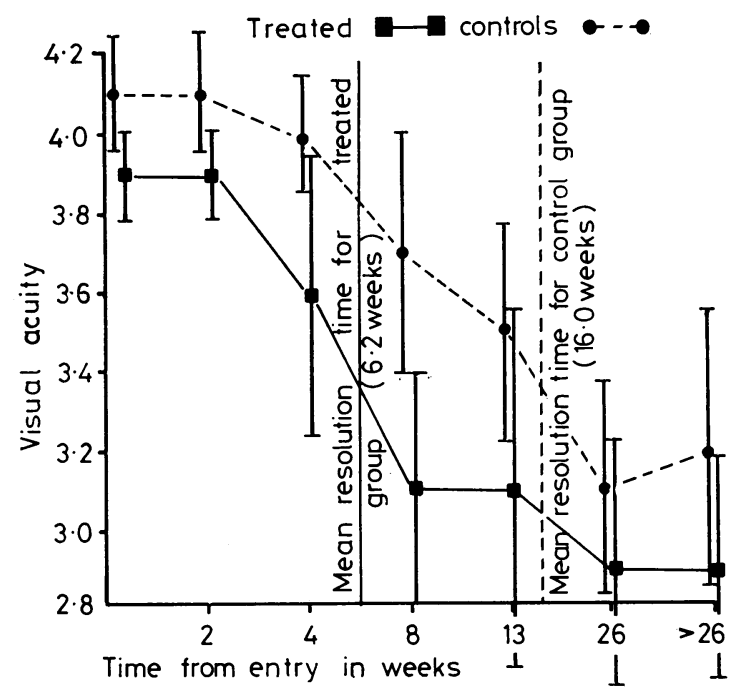

Fig. 1 Visual acuity; $1-6 / 4,2-6 / 5,3-6 / 6,4-6 / 9$, $5-6 / 12$. Graph showing the change in mean visual acuity of the treated and control groups from the time of entry into the trial

INFLUENCE OF OTHER FACTORS

No significant difference was found between the visual acuities or 100-hue scores of the 2 groups when they were subdivided according to the patient's age, size of serous detachment, distance of fluorescein leak from fovea, or evidence of pre-existing RPE disease.

\section{Discussion}

In the 2 groups of patients studied the results of visual acuity testing with the Snellen chart closely paralleled the Farnsworth Munsell 100-hue scores, and there was a clearly demonstrable deficit in affected compared with fellow eyes. However, in an individual case it was often difficult to detect this with the Snellen chart, while the sensitivity and measurability of the 100-hue test demonstrated it easily. This difference between the 2 methods of assessing visual morbidity was particularly clearly seen when comparing the final visual acuities of the 2 groups with those of the fellow eyes, when no significant difference was found in the Snellen acuities, while there was a marked difference in the 100-hue scores. This observation correlates well with the experience of many patients who complain of mild symptoms long after clinical signs have resolved completely.

The severity of the visual deficit associated with CSR is due in part to hypermetropia and astigmatism and in part to receptor dysfunction induced by the serous retinal detachment. Although visual acuity can usually be improved with correcting lenses, correction of the refractive error is neither an easy nor a practicable treatment, because the refractive change is at best temporary and at worst fluctuating. Treatment aimed at achieving retinal reapposition, thus correcting both the refractive error and restoring normal receptor function, may therefore be justified.

It is accepted that photocoagulation is effective in causing retinal reapposition, and the results of this study confirm that resolution occurs more rapidly after photocoagulation than it does in the absence of treatment. However, there seems little evidence to suggest that speedy reattachment of the retina confers any long-term visual advantage to the patient, since there was no significant difference in the final visual acuity between the treated and the untreated groups. Moreover, in no patient in this series did the visual acuity deteriorate to less than $6 / 12$, thus necessitating withdrawal from the trial. We therefore conclude that no patient lost vision because treatment was withheld, and persistence of serous retinal detachment did not lead to progressive receptor loss.

If our patients are representative of the disease as a whole, we are led to the conclusion that persistence of CSR does not lead to progressive deterioration of vision in those patients in whom the initial acuity is $6 / 12$ or better. This may be explained if the degree of visual deficit is determined early in the disease or if the disease we have been studying is different from that in which serous detachment is accompanied by severe visual loss. The hypothesis that poor visual recovery in CSR is due to progressive receptor cell attrition related to prolonged detachment has never been proved, and other factors such as choroidal or retinal pigment epithelial disease may be responsible.

If laser photocoagulation carried no visual morbidity it could still be argued that treatment is justifiable in all cases, but, however small the risk, potential complications are implicit in this form of treatment. There is danger of photocoagulating the fovea or of coagulating a macular venule or arteriole, resulting in foveal ischaemia and intraretinal fibrosis (Gass, 1972).

Finally, some authors have claimed that photocoagulation may precipitate a disciform lesion, with the serious threat to central vision that such a lesion carries (François et al., 1975; Schatz et al., 1977). While in most patients treatment with the argon laser can be safely undertaken, there is a small proportion in whom the proximity of the leaking point to the fovea or to the major blood vessels makes this procedure hazardous, and indirect 
treatment may not be a practicable alternative to photocoagulation of the leaking site itself.

We conclude from our observations that, while laser photocoagulation is a safe and satisfactory method of treatment in those cases with distressing symptoms, there is no evidence that it influences the final visual outcome in patients whose vision is $6 / 12$ or better and where there is no evidence of structural retinal damage. Treatment can be justified when the visual deficit affects the quality of life, particularly ability to work, but not on the basis of length of detachment alone. It should be possible to make a decision concerning treatment at the onset of the disease, as there is no evidence to suggest that the prognosis is influenced by the duration of serous detachment.

We are grateful to the consultant surgeons at Moorfields for allowing us to study their patients and to Professor A. C. Bird for his help and encouragement in the preparation of this paper. We thank Mr Hugh Donovan of the Computer Section, Institute of Ophthalmology, Judd Street, London $\mathrm{WCl}$, for the statistical analysis of our findings, $\mathrm{Mr} \mathrm{T}$. Tarrant for preparing the figure, and Miss Christine Giffen and Miss Heather Lucas for drafting the manuscript.

\section{References}

Amalric. (1974). Discussion of central serous retinopathy. Moderne Probleme der Ophthalmologie, 12, 361.

François, J., De Laey, J. J., Canıbie, E., Hanssens, M., and Victoria-Troncoso, V. (1975). Neovascularisation after argon laser photocoagulation of macular lesions. American Journal of Ophthalmology, 79, 206-210.

Gass, J. D. M. (1967). Pathogenesis of disciform detachment of the neuro-epithelium. II. Idiopathic central serous choroidopathy. American Journal of Ophthalmology, 63, 587-615.

Gass, J. D. M. (1972). Options in the treatment of macular diseases. Transactions of the Ophthalmological Societies of the United Kingdom, 92, 449-468.
Gass, J. D. M. (1974a). Discussion of central serous retinopathy. Modern Problems in Ophthalmology, 12, 361. Karger: Basel.

Gass, J. D. M. (1974b). Discussion of central serous retinopathy. Modern Problems in Ophthalmology, 12, 363. Karger: Basel.

Hogan, M. (1974). Discussion of central serous retinopathy. Modern Problems in Ophthalmology, 12, 360. Karger: Basel.

Klein, M. L., Van Buskirk, E. M., Friedman, E., Gragoudas, E., and Chandra, S. (1974). Experience with non-treatment of central serous choroidopathy. Archives of Ophthalmology, 91, 247-250.

Maumanee, A. E. (1965). Symposium, macular diseases, clinical manifestations. Transactions of the American Academy of Ophthalmology and Otolaryingology, 69, 605-613.

Nanjiani, M. (1977). Long term follow-up of central serous retinopathy. Transactions of the Ophthalmological Societies of the United Kingdom, 97, 656-661.

Peabody, R. R., Zwang, H. C., and Little, H. L. (1968). Treatment of persistent central serous retinopathy. Archives of Ophthalmology, 79, 166-169.

Schatz, H., Yanuzzi, L. A., and Gitter, K. A. (1977). Subretinal neovascularisation following argon laser photocoagulation treatment for central serous chorioretinopathy: complication or misdiagnosis. Transactions of the American Academy of Ophthalmology and Otolaryingology 83, 893-906.

Schoch, D. (1974). Discussion of central serous retinopathy. Moderne Probleme der Ophthalmologie, 12, 361.

Spalter, H. F. (1968). Photocoagulation of central serous retinopathy. Archives of Ophthalmology, 79, 247-253.

Watzke, Burton, T. C., and Leaverton, P. (1974). Ruby laser photocoagulation therapy of central serous retinopathy. A preliminary report. Modern Problems in Ophthalmology, 12, 242-246. Karger: Basel.

Wessing, A. (1971). Central serous retinopathy and related lesions. Modern Problems in Ophthalmology, 9, 148-151. Karger: Basel.

Wessing, A. (1974). Discussion of central serous retinopathy. Modern Problems in Ophthalmology, 12, 361. Karger: Basel.

Zweng, H. C. (1974). Discussion of central serous retinopathy. Modern Problems in Ophthalmology, 12, 363. Karger: Basel. 